\title{
The role of the clinical professions in the regulation of healthcare in England: walking the tightrope
}

\section{SUMMARY \\ The Royal Colleges and other profes- sional bodies could use their clinical expertise, authority and influence with clinicians to improve the quality of regulation of healthcare services. At present, their contribution to regulation in England is ad hoc and informal. Better engagement could increase the impact that professional \\ England's new regulator: the Care Quality Commission}

\author{
bodies have on patient care and \\ create a new role for them as arbiters \\ of quality in the organisation and \\ delivery of care. The requirement for \\ healthcare provider organisations to \\ register with the Care Quality \\ Commission from April 2010 and \\ the National Health Service Next \\ Stage Review have created an \\ opportunity for closer collaboration.
}

\begin{abstract}
However, there are problems that must be overcome. These include the ownership of information about the quality of care and the uses to which this is put. It would be self-defeating if closer working with the regulator undermined the trust that clinicians have in quality improvement work led by the professional bodies.
\end{abstract}

In April 2009 the Care Quality Commission became the regulator of health and social care services in England, and by April 2010 all healthcare providers must register with the new body. The Commission can suspend, cancel or impose conditions on registration, issue warnings and penalty notices or prosecute a trust. ${ }^{1}$ These enforcement powers suggest that we are entering an era of tougher regulation.

Under the Health and Social Care Act 2008, the Care Quality Commission 'must promote the effective co-ordination of reviews or assessments carried out by public bodies or other persons in relation to the carrying on of regulated activities'. This suggests that, as well as collaborating with other inspectorates such as Ofsted and HM Inspectorate of Prisons, the new regulator might wish to work with other bodies that use systematic approaches to evaluate the quality of services. This raises the question of the relationship between the activities of the Commission and the work of professional bodies that review and assess healthcare services, including the Royal College of Psychiatrists.

\section{Current role of Royal Colleges in assuring service quality}

The first of the stated objectives of the Royal College of Psychiatrists is to 'promote mental health by setting standards and promoting excellence in mental healthcare.'
Likewise, one of the aims of the Royal College of Physicians is to 'advise and work with government, the public, patients and other professions to improve health and healthcare ${ }^{3}$ and one of the aims of the Royal College of General Practitioners is to 'encourage and maintain the highest standards of general practice.' ${ }^{4}$ These broader objectives show that medical Royal Colleges see their role as extending beyond the narrower activities of setting clinical and educational standards for doctors. This is perhaps not surprising because the quality of care depends at least as much on how care is organised and delivered as on the skills and abilities of the individual clinicians - it is difficult even for a good doctor to provide good care consistently if they work in a bad service.

In keeping with this, some medical Royal Colleges have become involved actively in assuring the quality of healthcare provider organisations. The Royal College of Psychiatrists, through its Centre for Quality Improvement, manages a growing number of national networks that evaluate the quality of services using standards-based peer-review. The College's website provides more information about these quality improvement projects, including the standards that underpin them. ${ }^{5}$ On average, each specialist mental health trust in England participates in four of these networks; very few participate in none. The networks now cover most of the specialty areas of psychiatry and, increasingly, accredit the mental health services that participate. There are accreditation systems for electroconvulsive therapy (ECT) clinics, a range of psychiatric wards and therapeutic communities, and active plans to extend this to mental health liaison teams and memory services. 


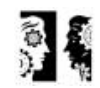

editorial
In other areas of healthcare, the Royal College of Pathologists established the Clinical Pathology Accreditation service in 1992, ${ }^{6}$ the Royal College of Radiologists has launched a Radiology Accreditation Programme, ${ }^{7}$ and the Royal College of Physicians has introduced a peer-review system for stroke services.

Medical Royal Colleges and other professional bodies also manage national clinical audits which, as well as measuring adherence to the National Institute for Health and Clinical Excellence guidance, collect highquality information about the performance of clinical teams, the process of care delivery and the structures required to deliver high-quality care. ${ }^{8}$ A number of these, such as the Myocardial Ischaemia National Audit Project established in 1999 by the Royal College of Physicians, ${ }^{9}$ have high rates of participation and so the data collected could be used to evaluate the relative quality of services nationally. The Royal College of Psychiatrists manages national audits that evaluate the pharmacological treatment of schizophrenia, the care of people with dementia in general hospitals and of the provision of psychological therapies for people with anxiety and depression. The last two audits focus as much on the process of care as they do on clinical decision-making.

\section{Links with regulation}

The principle that professionally led quality improvement should link with regulation was accepted before the establishment of the Care Quality Commission. The first objective of the Healthcare Concordat that the Academy of Medical Royal Colleges signed with the Care Quality Commission's predecessor, the Healthcare Commission, is to 'ensure that inspections are coordinated with other reviews and collections of data by: identifying opportunities for joint working, maximising opportunities for sharing information, sharing and unifying plans for inspection and review and developing a reliance on the results of work conducted by other competent bodies.'10 Some individual professional bodies, including the Royal College of Psychiatrists, took this one step further and signed a memorandum of understanding with the Healthcare Commission.

Despite the Concordat, there has so far been only limited joint working between professional bodies and the regulator in England. The Healthcare Commission required that provider services participate in a few of the national clinical audits and some National Health Service (NHS) trust boards have used their participation in professionally led quality improvement initiatives as evidence of compliance with the core Standards for Better Health. ${ }^{11}$ In mental health, accreditation by the ECT Accreditation Service was one of the information sources used by the Healthcare Commission to direct its assessment of mental health services against its core standards. However, these are exceptions. Most of the information collected by professionally led quality improvement networks and clinical audits has gone unused by the regulator.

\section{Benefits of closer collaboration}

So far, healthcare regulation in England has relied largely on self-assessment by service managers. This, coupled with preoccupation with centrally determined targets that are poor indicators of the quality of care, is part of an NHS culture that mitigates against full engagement by clinicians in quality improvement work. At its most extreme, this has contributed to the type of failings that happened in Mid Staffordshire. ${ }^{12}$ Lord Darzi, a surgeon who until recently was a Health Minister in England, has pledged to overturn this culture and to 'empower frontline staff to lead change that improves quality of care for patients.'.

The challenge for better regulation is that, despite investment in information management systems, there is little information collected routinely by NHS trusts that can be used to measure meaningfully the quality of the process of clinical care, the outcomes achieved or the level of patient satisfaction. In contrast, the information that is gathered by professionally led quality improvement networks and clinical audits is generally of a reasonable quality. This is because it is collected by, or its collection is overseen by, front-line clinicians who see how the information is relevant to them and their team and so have a personal interest in its accuracy and completeness. In short, the regulator needs the information that is collected by the professional bodies.

Better recognition by regulators would help the professional bodies. It would legitimise and formalise the role that some have taken on of defining and promoting the quality of healthcare services. Recognition would also increase participation in professionally led quality improvement programmes and so increase their value as sources of information.

For healthcare provider organisations, closer collaboration between the Colleges and the regulator would reduce the risk of duplication and increase positive engagement in the regulatory process by local clinicians. It might also help to orient the attention of the boards of NHS trusts to important and clinician-defined aspects of service delivery. This would help them to realise the vision set out by Lord Darzi and to better discharge their responsibilities in relation to clinical governance. These responsibilities are being more closely defined as a result of the policy focus on, for example, clinical metrics, payment by results, service line reporting and the provision of better information to the public to guide choice.

For patients and the public, collaboration could improve the quality of information about the quality of local healthcare services made available through various public portals. It would also increase the influence of patients and of the organisations that represent their interests in the process of standard setting and regulation. This is because patients and patient organisations play a prominent role in many professionally led quality improvement projects. 


\section{Risks and challenges of closer collaboration}

Professional bodies are not regulators. They are charities whose traditional purpose is to promote professional standards among doctors who are their members. However, as described earlier, they are already a part of the regulatory process. Also, professional bodies have in the past accredited or approved training schemes and it is envisaged that they will have a formal role in the regulation of healthcare professionals through their lead responsibility for recertification.

A more formal role in service regulation could create conflicts of interest. At its most basic, the problem is that professional bodies are membership organisations that rely heavily on membership subscriptions for their income. Membership is voluntary, although this may change when Colleges assume responsibilities for recertification. Should professional bodies put themselves in the position of directly or indirectly criticising their members? There are more subtle ways in which conflicts could arise. For example, professional bodies could be accused of having a vested interest in promoting the interests of their members, either locally within services or nationally as part of the normal process of lobbying.

The biggest obstacle to full collaboration is perhaps the issue of the ownership and use of the information collected by professional bodies. There is an expectation from government and from those that manage the NHS that data collected during quality improvement work should be placed in the public domain. This is for a range of purposes including local performance management, regulation, commissioning and informing patients and the public about the performance of their local services and, to some extent, of individual practitioners. The Bristol Inquiry catalysed this expectation and it is championed by Lord Darzi in his Next Stage Review..$^{13}$ In practice, there is great variation between professionally led quality improvement initiatives in the extent to which information is put into the public domain.

There appear to be a number of considerations that inhibit full disclosure. Some initiatives, particularly those established some years ago, have an understanding with the participating clinicians that release of data will be tightly controlled. This is sometimes a contractual commitment. There is also concern that data put into the public domain will in some way be misused or misinterpreted, for example by not properly addressing the complex issue of accounting for differences in case-mix. This could lead to clinicians or services being unfairly criticised, including by the media, and/or damaging unintended consequences such as clinicians becoming reluctant to take on 'difficult cases' for treatment. There are considerations that relate to the rights and safeguards under data protection legislation and under research ethics and research governance. These apply to both clinicians and to patients who both have, for example, a right to protection from information about them being used for a purpose other than that to which they have consented. There is sometimes a genuine concern that the effectiveness of quality improvement initiatives whose principal purpose is formative, that is promoting improvement through, for example, supporting professional development, could be undermined if the results are used for a summative purpose such as to inform the registration of services.

Finally, meaningful quality improvement depends totally on clinicians being honest about their performance. My experience is that this is invariably the case in quality improvement work led by the professions. There is the danger that the open disclosure of information about individual services or practitioners, without their full agreement, might fatally undermine the willingness of clinicians to provide the information, or might create incentives to game or otherwise to present the information in a misleading or overoptimistic light.

\section{Walking the tightrope}

The principal purpose of professionally led quality improvement work is to engage front-line clinicians in a robust and honest process to improve the quality of local services. All other uses of the information derived from these activities, no matter how important, should be considered secondary. To do otherwise would risk undermining the very attributes that ensure that the information is accurate, complete and meaningful. Having said that, all of this quality improvement work is at least partly funded by the NHS, directly or indirectly. The taxpayer therefore has a legitimate expectation that the information is used to maximum effect. In this era of transparent accountability, this includes its use to enable patients to make better informed choices.

The only way forward is for the professional bodies and the Care Quality Commission to negotiate how to maximise the impact of professionally led quality improvement work on the quality of care. The values that underpin this negotiation should be those of trust, equality and reciprocity. The Commission should recognise that professional bodies have a legitimate interest in service quality and that it is in the interests of English healthcare that the Commission promotes this role. On their part, professional bodies should be prepared to explore how the work that they lead can better support regulation. In June 2009, the government committed itself to support this when it stated that 'working with the Royal Colleges we will develop a consistent accreditation approach, beginning initially with a limited number of services and building up to extend this across every NHS service. The National Quality Board will play an important role in the development of this work.' ${ }^{14}$

\section{Declaration of interest}

None.

\section{References}

1 Care Quality Commission. Care Quality Commission Enforcement Policy. Care Quality Commission, 2009 
可

editorial
3 Royal College of Physicians. Our mission. Royal College of Physicians, 2009 (http:// www.rcplondon.ac.uk/ABOUTTHE-COLLEGE/MISSION/Pages/ Overview.aspx).

4 Royal College of General Practitioners. About the College. Royal College of General Practitioners, 2009 (http:// www.rcgp.org.uk/ about_the_college.aspx).

5 Royal College of Psychiatrists. College Centre for Quality Improvement (http://www. rcpsych.ac.uk/clinicalservice standards/centrefor qualityimprovement.aspx).
6 Royal College of Pathologists. Clinical PathologyAccreditation. CPA, 2002 (http://www. cpa-uk.co.uk/).

7 Royal College of Radiologists. Radiology accreditation programme. Royal College of Radiologists, 2009 (http:// accreditation.rcr.ac.uk/).

8 Healthcare Quality Improvement Partnership. Current audits. HQIP, 2009 (http://www.hqip.org.uk/ current-audits)

9 Royal College of Physicians. Myocardial Ischaemia National Audit Project (MINAP). Royal College of Physicians, 2009 (http://www.rcplondon.ac.uk/

CLINICAL-STANDARDS/

ORGANISATION/PARTNERSHIP/

Pages/MINAP-.aspx).

10 Concordat. Objective one inspections are coordinated with other reviews and collections of data. Concordat, 2009 (http:// www.concordat.org.uk/ objectives/objectiveone.cfm).

11 Department of Health. Standards for Better Health. Department of Health, 2004

Paul Lelliott Director, Royal College of Psychiatrists' Research and Training Unit, Standon House, 21 Mansell Street, London E1 8AA, email: plelliott@cru.rcpsych. ac.uk
12 Healthcare Commission nvestigation into Mid Staffordshire NHS Foundation Trust. Healthcare Commission, 2009

13 Department of Health. High Quality Care forAll: NHS Next Stage Review Final Report. TSO (The Stationery Office), 2008.

14 Department of Health. High Quality Care for All: Our Journey So Far. Department of Health, 2009. 